\section{Ab! Esses Anos Que Terminam Muito Rápido... (Cada Vez Mais!)}

E STAmos EM MEAdos DE DeZEMbro de 2005, praticamente às vésperas de um ano novo. Que, otimista como sempre, espero que seja melhor do que o que está acabando e que os anteriores, mas que, por intuição ou experiência, desconfio que vá ser igual, senão pior. E nem estou falando de política, economia ou meteorologia.

Refiro-me à incrível celeridade com que o ano irá esvanecer-se, pelo menos para aqueles que, como eu, estão entrando na emblemática "idade da razão"! Razão? Gozado, achava que tinha razão em tudo, mas isso quando jovem. Agora, mais "maduro", tenho a certeza - com a incômoda confirmação dos mais jovens - de que não tenho razão em quase nada. Então, por que "idade da razão”, nessa altura da vida? Talvez por essa característica crepuscular, de se permitir "filosofar" mais amiúde.

Como sempre faço na última edição do ano, apresento um resumo das atividades e do desempenho dos "Arquivos", e dedico esse editorial às pessoas que nos ajudaram a percorrer o caminho de maneira suave e a atravessar as barreiras e obstáculos do dia-a-dia sem maiores amarguras. Percalços sempre existirão, mas quem consegue viver sem um grande desafio pela frente? Aquela dose diária de adrenalina, como um bom café ou um cálice de vinho, é necessária - senão obrigatória -, para que a gente dê conta das vicissitudes da vida.

Assim, quero recordar que em 2005 produzimos, como temos feito desde 1998, seis edições regulares, sendo duas delas edições especiais, e mais seis suplementos dedicados a congressos da especialidade, que compuseram o volume 49 dos ABE\&M. As edições especiais ou temáticas foram dedicadas a duas áreas de importância inegável para o endocrinologista: "Determinação e Diferenciação do Sexo" e "Oncologia Endócrina”, tendo como editores convidados Andréa T. Maciel-Guerra e Gil GuerraJunior e Mauro A. Czepielewski e Jorge L. Gross, respectivamente. Agradeço mais uma vez a eles, e com renovado entusiasmo, pelas magníficas edições produzidas, sempre um sucesso junto aos leitores da revista. Aproveito, também, para provocá-los, caros leitores, com as edições especiais programadas para 2006: "Síndrome Metabólica" e "Osteoporose", a primeira a ser coordenada por Maria Teresa Zanella, Mário A. Saad e Sandra Roberta G. Ferreira e a segunda, por Francisco Bandeira, João Lindolfo C. Borges e John P. Bilezikian.

Em cada edição regular, cada vez mais volumosas, foi publicada uma média de 21 artigos, sendo 12 artigos originais, 3 revisões, 2 a 3 editoriais, 2 a 3 casos clínicos e 1 a 2 artigos nas sessões: Perspectivas e/ou Memórias. Junto com os 50 artigos das edições especiais, foram publicadas 1.010 páginas de texto científico no ano, um novo recorde. Esses números representam um incremento de $15 \%$ sobre o ano anterior.

As seis edições suplementares de 2005 foram dedicadas, respectivamente, ao $6^{\circ}$. COPEM - Congresso Paulista de Endocrinologia e
Professor Adjunto de Medicina,
Disciplina de Endocrinologia,
Departamento de Medicina,
Universidade Federal de
São Panlo, SP.
Editor-Chefe,
ABE\& $M$ 
Metabologia (vol. 49, no. 2, supl 1, Maio de 2005), ao ENDO Recife-2005 e $4^{\circ}$. COBEMOM - Congresso Brasileiro de Metabolismo Ósseo e Mineral (vol. 49, no. 3, supl 1, Junho de 2005), ao $1^{\circ}$. CBAEM - Congresso Brasileiro de Atualização em Endocrinologia e Metabologia (vol. 49, no. 3, supl 2, Julho de 2005), ao $11^{\circ}$. CBO - Congresso Brasileiro de Obesidade e V Congresso Latino Americano de Obesidade (vol. 49, no. 4, supl 1, Agosto de 2005), ao $6^{\circ}$. COBRAPEM - Congresso Brasileiro Pediátrico de Endocrinologia e Metabologia (vol. 49, no. 5, supl 1, Outubro de $2005)$ e ao $15^{\circ}$. Congresso da Sociedade Brasileira de Diabetes (vol. 49, no. 5, supl 2, Novembro de 2005). No total foram publicados, em igualmente 1.010 páginas de texto, mais de 1.200 resumos de trabalhos científicos e cerca de 200 resumos de palestras e conferências apresentadas ou proferidas nos vários eventos. Fica aqui, portanto, nossos agradecimentos àqueles que de alguma maneira contribuíram para produzir esse admirável material.

É indispensável enfatizar nos agradecimentos desse final de ano a valiosa participação dos nossos autores-colaboradores. O número de submissões, que em 2004 já tinha atingido 204 artigos, um valor histórico, nesse ano de 2005 manteve-se novamente em alta: até 10/12/2005, dia em que escrevo esse editorial, 230 artigos já haviam sido cadastrados, novamente uma estimativa de incremento de 15\%. Assim, nossos cordiais agradecimentos tanto àqueles colegas que têm mantido seu prestígio junto a revista, como àqueles novos que têm procurado nos $\mathrm{ABE} \& \mathrm{M}$ a melhor opção para suas publicações.

Um agradecimento especial aos nossos editorialistas de 2005, colegas sempre atenciosos e cordiais que, na maioria das vezes, são solicitados a trabalhar em prazo exíguo de tempo, mas jamais declinam do convite e, habitualmente, enviam seu material até antes do prazo. Quero cumprimentar e agradecer (em ordem cronológica da publicação) à: Andréa T. Maciel-Guerra, Gil Guerra-Junior, Ana Claudia Latronico, Valéria Guimarães, Marisa C. Coral, Regina do Carmo Silva, Margaret de Castro, Laura S. Ward, Durval Damiani, Mauro A. Czepielewski, Jorge L. Gross, Maria Lucia Fleiuss de Farias e Tânia Sanchez Bachega.

Por alguma razão, boa parte dos nossos tradicionais patrocinadores mostrou-se um pouco arredia em 2005. Vários declinaram de participar, sob pretextos muitas vezes injustificáveis, na medida em que a revista agora, após sua indexação internacional, tem muito maior destaque, visibilidade e penetração. No entanto, somos privilegiados de ainda poder contar com os auspícios de importantes empresas, às quais, mais uma vez, manifestamos publicamente nossos agradecimentos. São elas, em ordem alfabética: Abbott, CRIESP, Genzyme, Medley, Merck, Merck Sharp \& Dohme, Novo Nordisk, Pfizer, Roche, Sanofi-Aventis, Schering-Plough, Serono e Torrent.

Ao receber bimestralmente seu exemplar dos ABE\&M (intercalados pelos suplementos distribuídos nos vários Congressos da especialidade), os leitores muitas vezes não se dão conta do volumoso, incessante e crescente trabalho de bastidor que é necessário para se produzir a revista, englobando o cadastramento, organização e processamento dos manuscritos, as várias correções e revisões, a diagramação e editoração, os filmes e fotolitos e a impressão, o levantamento de patrocínio e contratos publicitários, a atualização cadastral, etiquetagem, envelopamento e distribuição, a contabilidade financeira da revista e do escritório, as reuniões deliberativas e a manutenção do cronograma e da ordem. É cada vez mais importante para os editores dos ABE\&M poder contar com o apoio e o prestígio dos anunciantes, que colocam inserções comerciais de seus produtos ou serviços e colaboram para o orçamento da revista. Os custos para produção de cada número são cada vez mais elevados, na medida que a revista à cada dia esta mais volumosa, implicando em maior custo de papel, diagramação, revisão e distribuição pelo correio.

Assim, sou profundamente grato à Blue Box, na pessoa da Estela Kater, que tem conseguido, a duras penas e com sacrifício pessoal, manter hoje a única fonte orçamentária dos $\mathrm{ABE} \& M$, pela venda de espaço para anúncios aos patrocinadores. A eficiência e pontualidade da equipe da TecArt, sob responsabilidade do Sr. Henrique Pereira, tem merecido durante todos esses anos de convívio amigável e harmonioso, o nosso permanente agradecimento. Agradeço, também, de coração, à Marina (Grámmata), pelas cuidadosas revisões e correções dos textos e galleys e à Roberta, pelo zelo, dedicação e cuidado com os assuntos secretariais.

Agradecimentos sinceros e cordiais são sempre devidos aos colegas que, nos bastidores, trabalham para garantir e melhorar cada vez mais a qualidade da revista, perscrutando manuscritos à cata de imperfeições e inadequações para sugerir mudanças aos 
autores e, por vezes, rejeitar manuscritos por insuficiência ou interpretação inadequada dos dados e por baixa prioridade, na medida em que o nobre e reduzido espaço da revista está cada vez mais concorrido. Nossos pareceristas ou revisores, com queiram, habitualmente prontificam-se a entregar suas revisões em tempos curtos, espremendo seu tempo e oferecendo sua disposição, conhecimento e experiência em benefício da revista e da Sociedade. O Conselho Editorial agradece a eles, porque seu trabalho determinado, gratuito e descompromissado é inestimável para os ABE\&M: foram solicitados a emitir pareceres durante 2005 (alguns deles por mais de 5 vezes no ano, quando a média é de 2,5 vezes ao ano) os seguintes colegas (em ordem alfabética de primeiro nome) aos quais somos muitíssimo gratos:

Adriana Costa e Forti, Alexander A.S. Jorge, Alfredo Halpern, Amanda V.L. de Athayde, Amélio F. Godoy Matos, Ana Claudia Latronico, Ana Claudia Ramalbo, Ana Luiza Maia, Ana Maria J. Lengyel, Ana Maria Lottenberg, André F. Reis, Andréa M. Britto Fioretti, Angela A.J. Reichelt, Antônio A. Barros, Antônio C. Bianco, Antonio C. Boschero, Antônio C. Lerário, Antônio H. Lancha Jr., Antônio R. Chacra, Antônio S. Tebexreni, Aurélio Borelli, Ayrton C. Moreira, Balduino Tschiedel, Berenice B. Mendonça, Bernardo L. Wajchenberg, Bernardo Liberman, Bruno Geloneze Neto, Carlos Alberto Longui, Carlos Eduardo Negrão, Carmen Pazos-Moura, César L. Boguszewski, César Y. Hayashida, Christine Hackel, Cláudio C. Albino, Claudio E. Kater, Cynthia M.A. Brandão, Dalva M. Rocha, Daniel Giannella Neto, Daniel Lerário, Décio Brunoni, Denise C. Coitinho, Denise P. Carvalho, Dolores Pardini, Domingos A. Malerbi, Doris Rosenthal, Durval Damiani, Éder C.R. Quintão, Edmund C. Barakat, Edna Nakandakare, Edna T. Kimura, Edson L. Arioli, Eduardo K. Tomimori, Eduardo P. Dias, Elaine M.F. Costa, Eliane B. Ribeiro, Fernando L. Alberto, Fernando S. Flexa Ribeiro Fo., Francisco Bandeira, Francisco J.A. de Paula, Frederico F. Ribeiro Maia, Gabriela L. Saraiva, Geraldo Medeiros-Neto, Gil Guerra-Junior, Gilberto Alonso, Gisab M. do Amaral, Giuseppe Repetto, Hans Graf, Helena Schmid, Hélio Bisi, Hélio Machado, Henrique de L. Suplicy, Hermelinda C. Pedrosa, Hilton Kuperman, Hugo R.K. Lisboa, Ieda T.N. Verreschi, Inai Novato Silva, Ivaldir S. Dalbosco, Ivani C. Trombeta, Ivo J.P. Arnbold, Jacqueline R. Araújo, Jader B. Ferreira, Jairo T. Hidal, João Carlos R. Dias, João $H$.
Romaldini, João Lindolfo C. Borges, João Roberto $M$. Martins, João Roberto Sá, Jorge L. Gross, José A. Sgarbi, José A. Sisson de Castro, José A.M. Marcondes, José B. Lopes de Faria, José B.C. Carvalheira, José Egídio P. de Oliveira, José Gilberto H. Vieira, Júlio Abucham Fo., Júlio Licinio, Júlio S. Marchini, Karla F.S. Mello, Laércio J. Franco, Laura S. Ward, Léa M.Z. Maciel, Leila M.B. Araújo, Leonardo M. Diniz, Licio A. Velloso, Lourenço Gallo Jr., Luciane Ribeiro-Neto, Luciano Giacaglia, Lucila L.K. Elias, Luis A. de Castro, Luis Eduardo P. Calliari, Luís Henrique S. Canani, Luiz Antonio N. Martins, Luiz de Lacerda Fo., Luiz Roberto Ramos, Luiza K. Matsumura, Malebranche B.C. da Cunba Neto, Marcello D. Bronstein, Márcia Nery, Márcio Abrahão, Márcio C. Mancini, Marcos A. Tambascia, Margaret C.S. Boguszewski, Margaret de Castro, Maria Adelaide A. Pereira, Maria Alice Bordallo, Maria Cândida V. Fragoso, Maria Elizabeth $R$. da Silva, Maria Helena Senger, Maria Inês Schmidt, Maria Lúcia C. Giannella, Maria Lúcia D'Arbo Alves, Maria Lúcia F. de Farias, Maria Odette R. Leite, Maria Teresa Zanella, Maria Tereza M. Baptista, Marília de B. Gomes, Marília M. Guimarães, Mário Antônio C. Filho, Mário J.A. Saad, Mário Vaisman, Marise Lazaretti-Castro, Mauro A. Czepielewski, Mauro Fisberg, Mauro Semer, Meyer Knobel, Miguel N. Hissa, Milena C. Fernandes-Caldato, Milton C. Foss, Mirela J. de Azevedo, Mirta Knopfelmacher, Moacir C. de Andrade Jr., Mônica R. Gadelha, Nélson Carvalhaes Neto, Nélson Rassi, Nicolau Lima Neto, Nina R.C. Musolino, Nuvarte Setian, Omar M. Hauache, Orsine Valente, Osmar Monte, Paulo H. Maccagnan, Paulo Tannús Jorge, Pedro Henrique S. Correa, Poli Mara Spritzer, Regina Célia S. Moisés, Regina do Carmo Silva, Reinaldo P. Furlanetto, Renata Azevedo, Ricardo de L. Zollner, Ricardo Meirelles, Roberto Bazotte, Rômolo Sandrini Neto, Rosa Ferreira dos Santos, Rosa Paula M. Biscolla, Rosalinda Y.A. de Camargo, Rosana Radominski, Rosana S. Cardoso Alves, Rosely Sichieri, Rui Curi, Rui M.B. Maciel, Ruth Clapauch, Ruy Lyra, Sami Liberman, Sandra M.F. Villares, Sandra R.G. Ferreira, Sebastião de Oliveira Jr., Sérgio Atala Dib, Sérgio P.A. Toledo, Silmara A. Oliveira Leite, Simão A. Lottenberg, Sonir R.R. Antonini, Sorabia Domenice, Suemi Marui, Táki A. Cordás, Tânia S. Bachega, Teresa C.A. Vieira, Themis Zelmanovitz, Thomaz R.P. Cruz, Turíbio Leite Barros Neto, Ubiratan F. Machado, Vaê Dichtchekenian, Vanda Jorgetti, Victor C. Pardini, Vinicius Nabime 
Brito, Walkyria G. Volpini, Walmir F. Coutinho, Walter Bloise, Walter C. de Lima, Walter J. Minicucci, Zuleika S.C. Halpern.

Deixo para o fim, para que adquira um tom de distinção, meu agradecimento especial a três colegas excepcionais: Ana Claudia Latronico, Mario Saad e Omar Hauache, co-editores que têm compartilhado comigo as responsabilidades e os encargos do Conselho Editorial dos $\mathrm{ABE} \& M$, aqui incluídos não apenas o processamento de trabalhos, mas também, a partilha de decisões da política editorial. A alegria e o alívio de poder dividir com eles esse mandato é enorme! Muitíssimo obrigado a vocês.

Por fim, aos nossos estimados colegas, leitores e estimuladores, quero desejar um Feliz Natal e um final de ano repleto de paz e com muita celebração. Que 2006 seja generoso e possa proporcionar a todos a realização de grandes e belos sonhos. 